\title{
Oscillations of Neutral Difference Equations of Second Order with Positive and Negative Coefficients
}

\author{
Hussain Ali Mohamad, Hala Majid Mohi \\ Dept. of Mathematics, College of Science for Women, University of Baghdad, Baghdad, Iraq \\ Email address: \\ Hussainmohamad22@gmail.com (H. A. Mohamad), hala.iraq96@yahoo.com (H. M. Mohi)
}

\section{To cite this article:}

Hussain Ali Mohamad, Hala Majid Mohi. Oscillations of Neutral Difference Equations of Second Order with Positive and Negative Coefficients. Pure and Applied Mathematics Journal. Vol. 5, No. 1, 2016, pp. 9-14. doi: 10.11648/j.pamj.20160501.12

\begin{abstract}
In this paper some necessary and sufficient conditions are obtained to guarantee the oscillation for bounded and all solutions of second order nonlinear neutral delay difference equations. In Theorem 5 and Theorem 8 , We have studied the oscillation criteria as well as the asymptotic behavior, where was established some sufficient conditions to ensure that every solution are either oscillates or $\left|y_{n}\right| \rightarrow \infty$ as $n \rightarrow \infty$. Examples are given to illustrate the obtained results.
\end{abstract}

Keywords: Oscillation, Neutral Difference Equations, Second Order Difference Equations

\section{Introduction}

In this paper the oscillation for bounded and all solutions of second order neutral delay difference equation with positive and negative coefficients:

$$
\Delta^{2}\left(y_{n}-p_{n} y_{n-m}\right)+q_{n} G\left(y_{n-k}\right)-r_{n} G\left(y_{n-l}\right)=f_{n}
$$

will be studied, where $\Delta$ is the forward difference operator, $q_{n}, r_{n}$ are nonnegative infinite sequences of real numbers and $f_{n}, p_{n}$, are infinite sequences of real numbers. $G \in(R, R)$ is function $y_{n} G\left(y_{n}\right)>0$. The purpose of this research is to obtain new sufficient conditions for the oscillation of all solutions of equation (1). The following assumptions are used:

$$
\begin{aligned}
& \left(\mathrm{H}_{1}\right) \sum_{j=n_{1}} \sum_{i=j+l-k}^{j-1} r_{i}<\infty ; \\
& \left(\mathrm{H}_{2}\right) \sum_{j=n_{1}}^{\infty} \sum_{i=j-l+k}^{j-1} q_{i}<\infty
\end{aligned}
$$

$\left(\mathrm{H}_{3}\right)$ There exists a sequence $\left\{F_{n}\right\}$ such that $\Delta^{2} F_{n}=f_{n}$ and $\lim _{n \rightarrow \infty} F_{n}=0$;

$$
\begin{aligned}
& \left(\mathrm{H}_{4}\right) G(y) \geq \beta_{1} y ; \\
& \left(\mathrm{H}_{5}\right) G(y) \leq \beta_{2} y .
\end{aligned}
$$

\section{Main Result}

The next results provide sufficient conditions for the oscillation of all bounded solutions of Eq. (1). For a simplicity set

$$
z_{n}=y_{n}-p_{n} y_{n-m}
$$

Let the sequence $w_{n}$ be defined as

$$
w_{n}=y_{n}-p_{n} y_{n-m}+\sum_{j=n}^{\infty} \sum_{i=j+l-k}^{j-1} r_{i} G\left(y_{i-l}\right)-F_{n}, k>l
$$

and the sequence $W_{n}$ be defined as

$$
W_{n}=y_{n}-p_{n} y_{n-m}-\sum_{j=n}^{\infty} \sum_{i=j-l+k}^{j-1} q_{i} G\left(y_{i-k}\right)-F_{n}, l>k
$$
184

The following theorem based on Theorem 7.6.1, [3] pp.

Theorem 1. ([3], pp. 184)

Assume that $\left\{p_{n}\right\}$ is a nonnegative sequence of real numbers and let $k$ be a positive integer. Suppose that

$$
\liminf _{n \rightarrow \infty} \sum_{i=n-k}^{n-1} p_{i}>\frac{k^{k+1}}{(k+1)^{k+1}}
$$

Then

i. The difference inequality

$$
y_{n+1}-y_{n}+p_{n} y_{n-k} \leq 0, \quad n=0,1,2, \cdots
$$

cannot have eventually positive solutions. 
ii. The difference inequality

$$
y_{n+1}-y_{n}+p_{n} y_{n-k} \geq 0, n=0,1,2, \cdots
$$

cannot have eventually negative solutions.

Theorem 2. ([12], pp.10) Let $x, y \in R$ then

(i) $x<y+\varepsilon$ for all $\varepsilon>0$ if and only if $x \leq y$.

(ii) $x>y-\varepsilon$ for all $\varepsilon>0$ if and only if $x \geq y$.

Theorem 3. Suppose that $p_{n} \geq 1$ is bounded $\left(r_{n+l-k}-\right.$ $\left.q_{n}\right) \leq 0$, let $\left(\mathrm{H}_{1}\right),\left(\mathrm{H}_{3}\right)$, and $\left(\mathrm{H}_{4}\right)$ hold, in addition to

$$
\begin{gathered}
\liminf _{n \rightarrow \infty} \sum_{\substack{j=n-(k-\rho-m) \\
i=j}}^{n-1} \frac{\left|r_{i+l-k}-q_{i}\right|}{p_{i-k+m}} \\
>\frac{(k-\rho-m)^{k-\rho-m+1}}{\beta_{1}(k-\rho-m+1)^{k-\rho-m+1}}, \\
k>\rho+m
\end{gathered}
$$

Then every bounded solution of equation (1) oscillates.

Proof. Assume for the sake of contradiction that $\left\{y_{n}\right\}$ be positive bounded solution of eq. (1) for $n \geq n_{0} \geq 0$, then from equations (1), (2) and (3) we obtain

$$
\Delta^{2} w_{n}=\left(r_{n+l-k}-q_{n}\right) G\left(y_{n-k}\right) \leq 0
$$

Hence, $\Delta w_{n}, w_{n}$ are monotone sequences. We claim that $\Delta w_{n}>0$ for $n \geq n_{1} \geq n_{0}$, otherwise, $\Delta w_{n}<0, n \geq n_{1}$, thus, $w_{n}<0$ and $w_{n} \rightarrow-\infty$ as $n \rightarrow \infty$. From (3) we get

$$
\begin{aligned}
& w_{n} \geq-p_{n} y_{n-m}-F_{n} \\
& \geq-p y_{n-m}-F_{n}, p_{n} \leq p
\end{aligned}
$$

then $y_{n} \rightarrow \infty$ as $n \rightarrow \infty$, which is a contradiction. Hence our claim is established. We have two cases for $n \geq n_{2} \geq n_{1}$ :

Case 1: $w_{n}>0, \Delta w_{n}>0, \Delta^{2} w_{n} \leq 0$; Case 2: $w_{n}<$ $0, \Delta w_{n}>0, \Delta^{2} w_{n} \leq 0$

Case 1: $w_{n}>0, \Delta w_{n}>0, \Delta^{2} w_{n} \leq 0$, then there exists $\gamma>0$ such that $w_{n} \geq \gamma>0$ for $n \geq n_{2} \geq n_{1}$. Since $y_{n}$ is bounded, let $\liminf _{n \rightarrow \infty} y_{n}=h_{*} h_{*} \geq 0$, so there exists a subsequence $\left\{n_{j}\right\}$ of $\{n\}$ such that $\lim _{j \rightarrow \infty} n_{j}=\infty$, $\lim _{j \rightarrow \infty} y_{n_{j}}=h_{*}$. From (3) we get

$$
\begin{gathered}
y_{n_{j}}=w_{n_{j}}+p_{n_{j}} y_{n_{j}-m}-\sum_{s=n_{j}}^{\infty} \sum_{i=s+l-k}^{s-1} r_{i} G\left(y_{i-l}\right)+F_{n_{j}} \\
y_{n_{j}} \geq \gamma+p_{n_{j}} y_{n_{j}-m}-\delta_{2} \sum_{s=n_{j}}^{\infty} \sum_{i=s+l-k}^{s-1} r_{i}+F_{n_{j}}, G\left(y_{n}\right) \leq \delta_{2} \\
y_{n_{j}}>\gamma+y_{n_{j}-m}-\varepsilon
\end{gathered}
$$

Since $\varepsilon$ is arbitrary, by Theorem 2 , it follows that for sufficiently large $j$ we get

$$
y_{n_{j}} \geq \gamma+y_{n_{j}-m}
$$

As $j \rightarrow \infty$, it follows that $h_{*} \geq \gamma+h_{*}$ which is a contradiction.

Case 2: $w_{n}<0, \Delta w_{n}>0, \Delta^{2} w_{n} \leq 0$. By taking the summation of both sides of (6) from $n$ to $n+\rho, 0<\rho<$ $k-m$, we get

$$
\begin{gathered}
\Delta w_{n+1}-\Delta w_{n}=\sum_{i=n}^{n+\rho}\left(r_{i+l-k}-q_{i}\right) G\left(y_{i-k}\right) \\
-\Delta w_{n} \leq \beta_{1} \sum_{i=n}^{n+\rho}\left(r_{i+l-k}-q_{i}\right) y_{i-k} \\
\Delta w_{n} \geq \beta_{1} \sum_{i=n}^{n+\rho}\left|r_{i+l-k}-q_{i}\right| y_{i-k}
\end{gathered}
$$

From (3) we get

$$
\begin{gathered}
w_{n}=y_{n}-p_{n} y_{n-m}+\sum_{j=n}^{\infty} \sum_{i=j+l-k}^{j-1} r_{i} G\left(y_{i-l}\right)-F_{n} \\
\geq-p_{n} y_{n-m}-F_{n} \\
w_{n}>-p_{n} y_{n-m}-\varepsilon, \varepsilon>0
\end{gathered}
$$

Since $\varepsilon$ is arbitrary, it follows that

$$
\begin{aligned}
w_{n} & \geq-p_{n} y_{n-m} \\
y_{n-m} & \geq-\frac{1}{p_{n}} w_{n} \\
y_{n-k} & \geq-\frac{1}{p_{n-k+m}} w_{n-k+m}
\end{aligned}
$$

Substituting (8) in (7) to obtain

$$
\begin{gathered}
\Delta w_{n} \geq-\beta_{1} \sum_{i=n}^{n+\rho} \frac{\left|r_{i+l-k}-q_{i}\right|}{p_{i-k+m}} w_{i-k+m} \\
\Delta w_{n}+\beta_{1} \sum_{i=n}^{n+\rho} \frac{\left|r_{i+l-k}-q_{i}\right|}{p_{i-k+m}} w_{n-(k-\rho-m)} \geq 0
\end{gathered}
$$

By theorem 1-ii and in virtue of (5), it follows that the last inequality cannot have eventually negative solution, which is a contradiction.

Example 4. Consider the difference equations

$$
\begin{gathered}
\Delta^{2}\left(y_{n}-\left(1+\left(\frac{1}{2}\right)^{n}\right) y_{n-1}\right)+\frac{27}{32} y_{n-3}-\frac{25}{32}\left(\frac{1}{2}\right)^{n} y_{n-2} \\
=\left(\frac{1}{2}\right)^{n+3}\left(-\frac{1}{2}\right)^{n}
\end{gathered}
$$

where $\mathrm{m}=1, \mathrm{k}=3, \mathrm{l}=2, \rho=1, p_{n}=1+\left(\frac{1}{2}\right)^{n}, q_{n}=\frac{27}{32}$, $r_{n}=\frac{25}{32}\left(\frac{1}{2}\right)^{n}, G\left(y_{n}\right)=y_{n}, \beta_{1}=1, f_{n}=\left(\frac{1}{2}\right)^{n+3}\left(-\frac{1}{2}\right)^{n}$

- $\sum_{j=n_{1}}^{\infty} \sum_{i=j+l-k}^{j-1} r_{i}=\frac{25}{32} \sum_{j=n_{1}}^{\infty}\left(\frac{1}{2}\right)^{i-1}=\frac{25}{16}<\infty$

- $p_{n}=1+\left(\frac{1}{2}\right)^{n} \geq 1$.

- $r_{n+l-k}-q_{n}=25\left(\frac{1}{2}\right)^{n+3}-\frac{27}{32}<0, n \geq 1$.

- $\liminf _{n \rightarrow \infty} \sum_{j=n-1}^{n-1} \sum_{i=j}^{j+1} \frac{\left|r_{i+l-k}-q_{i}\right|}{p_{i-k+m}}=$ $\lim _{n \rightarrow \infty} \sum_{n-1}^{n-1} \sum_{i=j}^{j+1} \frac{\frac{27}{32}-25\left(\frac{1}{2}\right)^{i+3}}{1+\left(\frac{1}{2}\right)^{i-2}}=\frac{27}{16}>\frac{1}{4}$ 
By Theorem 3, it follows that every bounded solution of (E1) oscillates, for instance $y_{n}=\left(-\frac{1}{2}\right)^{n}$ is such a solution.

Theorem 5. Suppose that $p_{n} \leq p<1,\left(r_{n+l-k}-q_{n}\right) \leq 0$, $\left(\mathrm{H}_{1}\right),\left(\mathrm{H}_{3}\right)-\left(\mathrm{H}_{5}\right)$ hold, in addition to

$$
\begin{gathered}
\sum_{i=n_{0}}^{\infty} q_{i}=\infty, n_{0} \geq 0 \\
\liminf _{n \rightarrow \infty} \sum_{j=n-(k-\rho-m)}^{n-1} \sum_{i=j}^{j+\rho}\left|r_{i+l-k}-q_{i}\right| \\
>\frac{p(k-\rho-m)^{k-\rho-m+1}}{\beta_{1}(k-\rho-m+1)^{k-\rho-m+1}}, k>\rho+m
\end{gathered}
$$

Then every solution $\left\{y_{n}\right\}$ of equation (1) either oscillates or $\left|y_{n}\right| \rightarrow \infty$ as $n \rightarrow \infty$.

Proof. For the sake of contradiction, assume that $\left\{y_{n}\right\}$ be an eventually positive solution of eq. (1), then from equations (1), (2) and (3) it follows that (6) hold, that is

$$
\Delta^{2} w_{n}=\left(r_{n+l-k}-q_{n}\right) G\left(y_{n-k}\right) \leq 0
$$

Hence $\Delta w_{n}, w_{n}$ are monotone sequences. If $\Delta w_{n}<0$ for $n \geq n_{1} \geq n_{0}$, thus $w_{n}<0$ and $w_{n} \rightarrow-\infty$ as $n \rightarrow \infty$. From (3) we obtain

$$
\begin{gathered}
w_{n}=y_{n}-p_{n} y_{n-m}+\sum_{j=n}^{\infty} \sum_{i=j+l-k}^{j-1} r_{i} G\left(y_{i-l}\right)-F_{n}, \\
w_{n} \geq-p_{n} y_{n-m}-F_{n} \geq-p y_{n-m}-F_{n}
\end{gathered}
$$

which implies that $y_{n} \rightarrow \infty$ as $n \rightarrow \infty$.

If $\Delta w_{n}>0$ for $n \geq n_{1} \geq n_{0}$, we have two cases to consider for $n \geq n_{2} \geq n_{1}$ :

Case 1: $w_{n}>0, \Delta w_{n}>0, \Delta^{2} w_{n} \leq 0$; Case 2: $w_{n}<$ $0, \Delta w_{n}>0, \Delta^{2} w_{n} \leq 0$

Case 1: $w_{n}>0, \Delta w_{n}>0, \Delta^{2} w_{n} \leq 0$. Then $\lim _{n \rightarrow \infty} w_{n}=$ $L$, where $0<L \leq \infty$.

If $L=\infty$, From (3) we get

$$
\begin{gathered}
w_{n}=y_{n}-p_{n} y_{n-m}+\sum_{j=n}^{\infty} \sum_{i=j+l-k}^{j-1} r_{i} G\left(y_{i-l}\right)-F_{n} \\
w_{n} \leq y_{n}+\sum_{j=n}^{\infty} \sum_{i=j+l-k}^{j-1} r_{i} G\left(y_{i-l}\right)-F_{n}
\end{gathered}
$$

which implies that $\lim _{n \rightarrow \infty} y_{n}=\infty$, otherwise if $y_{n}$ is bounded it follows from the last inequality $w_{n}<y_{n}+\varepsilon$, which is a contradiction.

If $0<L<\infty$, then there exists $\gamma>0$ such that $w_{n} \geq \gamma>$ 0 , for $n \geq n_{2}$. If $\lim _{n \rightarrow \infty} y_{n}<\infty$, then from (3) we get

$$
\begin{gathered}
w_{n} \leq y_{n}+\beta_{2} \sum_{j=n}^{\infty} \sum_{i=j+l-k}^{j-1} r_{i} y_{i-k}-F_{n} \\
\leq y_{n}+\beta_{2} \delta_{1} \sum_{j=n}^{\infty} \sum_{i=j+l-k}^{j-1} r_{i}-F_{n}, y_{n} \leq \delta_{1} \\
w_{n}<y_{n}+\varepsilon, \varepsilon>0
\end{gathered}
$$

$\varepsilon$ is arbitrary, so for sufficiently large $n$ we get

$$
y_{n} \geq w_{n} \geq \gamma>0
$$

By taking the summation of both sides of (6) from $n_{2}$ to $n-1$, it follows that

$$
\begin{gathered}
\sum_{i=n_{2}}^{n-1} \Delta w_{i+1}-\Delta w_{i}=\sum_{i=n_{2}}^{n-1}\left(r_{i+l-k}-q_{i}\right) G\left(y_{i-k}\right) \\
\Delta w_{n}-\Delta w_{n_{2}} \leq \beta_{1} \sum_{i=n_{2}}^{n-1}\left(r_{i+l-k}-q_{i}\right) y_{i-k} \\
\leq \gamma \beta_{1} \sum_{i=n_{2}}^{n-1}\left(r_{i+l-k}-q_{i}\right) \\
-w_{n}-\Delta w_{n_{2}} \leq \gamma \beta_{1} \sum_{i=n_{2}}^{n-1}\left(r_{i+l-k}-q_{i}\right)
\end{gathered}
$$

In virtue of (9) the last inequality implies that $\lim _{n \rightarrow \infty} w_{n}=\infty$. Leads to a contradiction.

Case 2: $w_{n}<0, \Delta w_{n}>0, \Delta^{2} w_{n} \leq 0$. In this case $w_{n}$ is bounded, we claim that $y_{n}$ is bounded, otherwise there exists a subsequence $\left\{n_{j}\right\}$ of $\{n\}$ such that $\lim _{j \rightarrow \infty} n_{j}=\infty$, $\lim _{j \rightarrow \infty} y_{n_{j}}=\infty$ and $y_{n_{j}}=\max \left\{y_{n}: n_{2} \leq n \leq n_{j}\right\}$, from (3) we get

$$
\begin{gathered}
w_{n_{j}} \geq y_{n_{j}}-p_{n_{j}} y_{n_{j}-m}-F_{n_{j}} \\
w_{n_{j}} \geq(1-p) y_{n_{j}}-F_{n_{j}}
\end{gathered}
$$

which implies that $\lim _{j \rightarrow \infty} w_{n_{j}}=\infty$, a contradiction.

By taking the summation of both sides of (6) from $n$ to $n+\rho, \rho<k-m$, it follows that

$$
\begin{gathered}
\sum_{i=n}^{n+\rho} \Delta w_{i+1}-\Delta w_{i}=\sum_{i=n}^{n+\rho}\left(r_{i+l-k}-q_{i}\right) G\left(y_{i-k}\right)-\Delta w_{n} \leq \\
\beta_{1} \sum_{i=n}^{n+\rho}\left(r_{i+l-k}-q_{i}\right) y_{i-k}
\end{gathered}
$$

From (3) we get

$$
\begin{gathered}
w_{n}=y_{n}-p_{n} y_{n-m}+\sum_{j=n}^{\infty} \sum_{i=j+l-k}^{j-1} q_{i} G\left(y_{i-k}\right)-F_{n} \\
\geq-p_{n} y_{n-m}-F_{n} \\
w_{n}>-p y_{n-m}-\varepsilon, \varepsilon>0
\end{gathered}
$$

Since $\varepsilon$ is arbitrary, it follows that for sufficiently large $n$ :

$$
y_{n} \geq \frac{-1}{p} w_{n+m}
$$

Substituting the last inequality in (11) we obtain

$$
-\Delta w_{n} \leq \frac{-\beta_{1}}{p} \sum_{i=n}^{n+\rho}\left(r_{i+l-k}-q_{i}\right) w_{i+m-k}
$$




$$
\begin{gathered}
\leq \frac{\beta_{1}}{p}\left(\sum_{i=n}^{n+\rho}\left|r_{i+l-k}-q_{i}\right|\right) w_{n+\rho+m-k} \\
\Delta w_{n}+\frac{\beta_{1}}{p}\left(\sum_{i=n}^{n+\rho}\left|r_{i+l-k}-q_{i}\right|\right) w_{n-(k-\rho-m)} \geq 0
\end{gathered}
$$

By theorem 1-ii and in virtue of (10), it follows that the last inequality cannot have eventually negative solution, which is a contradiction.

In the next theorem we will use the sequence $W_{n}$ already defined in (4).

Theorem 6. Suppose that $p_{n} \leq 1,\left(r_{n}-q_{n-l+k}\right) \geq 0$, $\left(\mathrm{H}_{2}\right)-\left(\mathrm{H}_{3}\right)$, and $\left(\mathrm{H}_{5}\right)$ hold, in addition to

$$
\begin{gathered}
\liminf _{n \rightarrow \infty} \sum_{j=n-(l-\rho)}^{n-1} \sum_{i=j}^{j+\rho}\left(r_{i}-q_{i-l+k}\right)>\frac{(l-\rho)^{l-\rho+1}}{\beta_{1}(l-\rho+1)^{l-\rho+1}} \\
l>\rho
\end{gathered}
$$

Then every bounded solution of equation (1) oscillates.

Proof. For the sake of contradiction, assume that $\left\{y_{n}\right\}$ be an eventually positive bounded solution of eq. (1), then from equations (1), (2) and (4) we obtain

$$
\Delta^{2} W_{n}=\left(r_{n}-q_{n-l+k}\right) G\left(y_{n-l}\right) \geq 0
$$

Hence, $\Delta W_{n}, W_{n}$ are monotone sequences, we claim that $\Delta W_{n}<0$ for $n \geq n_{1} \geq n_{0}$, otherwise $\Delta W_{n}>0$ for $n \geq n_{1}$, hence $W_{n}>0$ and $W_{n} \rightarrow \infty$ as $n \rightarrow \infty$. Let $y_{n} \leq \delta_{1}$, then $G\left(y_{n}\right) \leq \beta_{2} \delta_{1}=\delta_{2}$, where $\delta_{1}, \delta_{2}$ are positive constants. From (4) we obtain

$$
\begin{gathered}
W_{n}=y_{n}-p_{n} y_{n-m}-\sum_{j=n}^{\infty} \sum_{i=j-l+k}^{j-1} q_{i} G\left(y_{i-k}\right)-F_{n} \\
W_{n} \leq y_{n}-F_{n}
\end{gathered}
$$

which implies that $y_{n} \rightarrow \infty$ as $n \rightarrow \infty$, which is a contradiction. Our claim has been established, then it remains to consider two possible cases for the existence of nonoscillatory solution of eq. (1) for $n \geq n_{2} \geq n_{1}$ :

Case 1: $W_{n}<0, \Delta W_{n}<0, \Delta^{2} W_{n} \geq 0$; Case 2: $W_{n}>$ $0, \Delta W_{n}<0, \Delta^{2} W_{n} \geq 0$;

Case 1: $W_{n}<0, \Delta W_{n}<0, \Delta^{2} W_{n} \geq 0$. Then there exists $\gamma<0$ such that, $W_{n} \leq \gamma<0$, for $n \geq n_{2}$. Since $y_{n}$ is bounded, let $\limsup _{n \rightarrow \infty} y_{n}=h^{*}, h^{*} \geq 0$ so there exists a sequence $\left\{n_{j}\right\}$ such that $\lim _{j \rightarrow \infty} n_{j}=\infty, \lim _{j \rightarrow \infty} y_{n_{j}}=h^{*}$. From (4) we get

$$
\begin{gathered}
y_{n}=W_{n}+p_{n} y_{n-m}+\sum_{j=n}^{\infty} \sum_{i=j+k-l}^{j-1} q_{i} G\left(y_{i-k}\right)+F_{n} \\
y_{n} \leq \gamma+p_{n} y_{n-m}+\delta_{2} \sum_{j=n}^{\infty} \sum_{i=j-l+k}^{j-1} q_{i}+F_{n} \\
y_{n}<\gamma+y_{n-m}+\varepsilon, \varepsilon>0
\end{gathered}
$$

Since $\varepsilon$ is arbitrary, then by theorem 2.2 , it follows for sufficiently large $j$ that:

$$
y_{n_{j}} \leq \gamma+y_{n_{j}-m}
$$

as $j \rightarrow \infty$, we get from the last inequality $h^{*} \leq \gamma+h^{*}$ which is a contradiction.

Case 2: $W_{n}>0, \Delta W_{n}<0, \Delta^{2} W_{n} \geq 0$. By taking the summation of both sides of (13) from $n$ to $n+\rho, \rho<l$ it follows that

$$
\begin{gathered}
\Delta W_{n+1}-\Delta W_{n}=\sum_{i=n}^{n+\rho}\left(r_{i}-q_{i-l+k}\right) G\left(y_{n-l}\right)-\Delta W_{n} \\
\geq \beta_{1} \sum_{i=n}^{n+\rho}\left(r_{i}-q_{i-l+k}\right) y_{i-l}
\end{gathered}
$$

From (4) we get

$$
\begin{aligned}
& y_{n}=W_{n}+p_{n} y_{n-m}+\sum_{j=n}^{\infty} \sum_{i=j-l+k}^{j-1} q_{i} G\left(y_{i-k}\right)+F_{n} \\
& \geq W_{n}+F_{n} \\
& y_{n}>W_{n}-\varepsilon, \varepsilon>0
\end{aligned}
$$

Since $\varepsilon$ is arbitrary, it follows that

$$
y_{n} \geq W_{n}
$$

Substituting the last inequality in (14) we obtain

$$
\begin{gathered}
-\Delta W_{n} \geq \beta_{1} \sum_{i=n}^{n+\rho}\left(r_{i}-q_{i-l+k}\right) W_{i-l} \\
-\Delta W_{n} \geq \beta_{1}\left(\sum_{i=n}^{n+\rho}\left(r_{i}-q_{i-l+k}\right)\right) W_{n+\rho-l} \\
\Delta W_{n}+\beta_{1}\left(\sum_{i=n}^{n+\rho}\left(r_{i}-q_{i-l+k}\right)\right) W_{n-(l-\rho)} \leq 0
\end{gathered}
$$

By Theorem 1-i, and in virtue of (12), it follows that the last inequality cannot have eventually positive solution, which is a contradiction.

Example 7. Consider the difference equation

$$
\begin{aligned}
\Delta^{2}\left(y_{n}\right. & \left.-\left(1+\left(\frac{1}{e}\right)^{n}\right) y_{n-m}\right)+\left(\frac{1}{e}\right)^{n}\left(-\frac{1}{e}\right)^{n-1} \\
- & {\left[e^{-4}\left(1+2 e+e^{2}\right)\right]\left(-\frac{1}{e}\right)^{n-2} } \\
= & \left(e^{-3}+2 e^{-1}\right)\left[\left(\frac{1}{e}\right)^{n}\left(-\frac{1}{e}\right)^{n}\right]
\end{aligned}
$$

where $k=1, m=1, l=2, \rho=1, \beta_{1}=1, p_{n}=1+\left(\frac{1}{e}\right)^{n}, q_{n}=$ $\left(\frac{1}{e}\right)^{n}$

$$
r_{n}=e^{-4}\left(1+2 e+e^{2}\right)
$$




$$
f_{n}=\left(e^{-3}+2 e^{-1}\right)\left[\left(\frac{1}{e}\right)^{n}\left(-\frac{1}{e}\right)^{n}\right], G\left(y_{n}\right)=y_{n}
$$

- $\sum_{j=n_{1}}^{\infty} \sum_{i=j-l+k}^{j-1} q_{i}=\sum_{j=n_{1}}^{\infty} \sum_{i=j-1}^{j-1}\left(\frac{1}{e}\right)^{i}=$ $\sum_{j=n_{1}}^{\infty}\left(\frac{1}{e}\right)^{j-1}=e \sum_{j=n_{1}}^{\infty}\left(\frac{1}{e}\right)^{j}<\infty$

- $p_{n}=\left(\frac{1}{e}\right)^{n}<1, n>0$

- $r_{n}-q_{n-l+k}=e^{-4}\left(1+2 e+e^{2}\right)-\left(\frac{1}{e}\right)^{n-1}=$ $e^{-4}\left(1+2 e+e^{2}\right)-e\left(\frac{1}{e}\right)^{n}>0, n \geq 0$.

- $\liminf _{n \rightarrow \infty} \sum_{j=n-(l-\rho)}^{n-1} \sum_{i=j}^{j+\rho}\left(r_{i}-\right.$

$$
\begin{aligned}
& \left.q_{i-l+k}\right)=\liminf _{n \rightarrow \infty} \sum_{j=n-1}^{n-1} \sum_{i=j}^{j+1} e^{-4}\left(1+2 e+e^{2}\right)- \\
& \left(\frac{1}{e}\right)^{i-1}=2 e^{-4}\left(1+2 e+e^{2}\right)>\frac{1}{4}
\end{aligned}
$$

By theorem 5, every bounded solution of (E2) oscillates, for instance $y_{n}=\left(-\frac{1}{e}\right)^{n}$ is such a solution.

Theorem 8. Suppose that $p_{n} \leq p,\left(r_{n}-q_{n-l+k}\right) \geq 0,\left(\mathrm{H}_{2}\right)$ $-\left(\mathrm{H}_{5}\right)$ hold, in addition to (12) and

$$
\sum_{i=n_{1}}^{\infty} r_{i}=\infty, n_{0} \geq 0
$$

Then every solution $\left\{y_{n}\right\}$ of equation (1) either oscillates or $\left|y_{n}\right| \rightarrow \infty$ as $n \rightarrow \infty$.

proof. For the sake of contradiction, assume that $\left\{y_{n}\right\}$ be an eventually positive solution of eq. (1), then from equations (1), (2) and (4) it follows that (13) hold, that is

$$
\Delta^{2} W_{n}=\left(r_{n}-q_{n-l+k}\right) G\left(y_{n-l}\right) \geq 0
$$

Hence, $\Delta W_{n}, W_{n}$ are monotone sequences. If $\Delta W_{n}>0$ for $n \geq n_{1} \geq n_{0}$, thus $W_{n}>0$ and $W_{n} \rightarrow \infty$ as $n \rightarrow \infty$. From (4) we obtain

$$
\begin{gathered}
W_{n}=y_{n}-p_{n} y_{n-m}-\sum_{j=n}^{\infty} \sum_{i=j-l+k}^{j-1} q_{i} G\left(y_{i-k}\right)-F_{n} \\
W_{n} \leq y_{n}-F_{n}
\end{gathered}
$$

which implies that $y_{n} \rightarrow \infty$ as $n \rightarrow \infty$.

If $\Delta W_{n}<0$ for $n \geq n_{1} \geq n_{0}$ we have two cases to consider for $n \geq n_{2} \geq n_{1}$ :

Case 1: $W_{n}<0, \Delta W_{n}<0, \Delta^{2} W_{n} \geq 0$; Case 2: $W_{n}>$ $0, \Delta W_{n}<0, \Delta^{2} W_{n} \geq 0$.

Case 1: $W_{n}<0, \Delta W_{n}<0, \Delta^{2} W_{n} \geq 0$. Then $\lim _{n \rightarrow \infty} W_{n}=L$, where $-\infty \leq L<0$.

If $L=-\infty$, From (4) we get

$$
\begin{gathered}
W_{n}=y_{n}-p_{n} y_{n-m}-\sum_{j=n}^{\infty} \sum_{i=j-l+k}^{j-1} q_{i} G\left(y_{i-k}\right)-F_{n} \\
W_{n} \geq-p y_{n-m}-\beta_{2} \sum_{j=n}^{\infty} \sum_{i=j-l+k}^{j-1} q_{i} y_{i-k}-F_{n}
\end{gathered}
$$

which implies that $\lim _{n \rightarrow \infty} y_{n}=\infty$.

If $-\infty<L<0$, then there exists $\gamma<0$ such that $W_{n} \leq$ $\gamma<0$, for $n \geq n_{2}$. If $\lim _{n \rightarrow \infty} y_{n}<\infty$. From (4) we get

$$
\begin{gathered}
W_{n} \geq-p_{n} y_{n-m}-\beta_{2} \sum_{j=n}^{\infty} \sum_{i=j-l+k}^{j-1} q_{i} y_{i-k}-F_{n} \\
W_{n}>-p_{n} y_{n-m}-\varepsilon, \varepsilon>0 \\
\gamma \geq W_{n} \geq-p_{n} y_{n-m} \\
y_{n-m} \geq-\frac{\gamma}{p}
\end{gathered}
$$

By taking summation to both sides of (13) from $n_{2}$ to $n-1$, it follows that

$$
\begin{gathered}
\sum_{i=n_{2}}^{n-1} \Delta W_{i+1}-\Delta W_{i}=\sum_{i=n_{2}}^{n-1}\left(r_{i}-q_{i-l+k}\right) G\left(y_{i-l}\right) \\
\Delta W_{n}-\Delta W_{n_{2}} \geq \beta_{1} \sum_{i=n_{2}}^{n-1}\left(r_{i}-q_{i-l+k}\right) y_{i-l} \\
\geq-\frac{\gamma \beta_{1}}{p} \sum_{i=n_{2}}^{n-1}\left(r_{i}-q_{i-l+k}\right) \\
-W_{n}-\Delta W_{n_{2}} \geq \beta_{1} \sum_{i=n_{2}}^{n-1}\left(r_{i}-q_{i-l+k}\right) y_{i-l} \\
\geq-\frac{\gamma \beta_{1}}{p} \sum_{i=n_{2}}^{n-1}\left(r_{i}-q_{i-l+k}\right)
\end{gathered}
$$

In virtue of (12) the last inequality implies that $\lim _{n \rightarrow \infty} W_{n}=-\infty$. Leads to a contradiction.

Case 2: $W_{n}>0, \Delta W_{n}<0, \Delta^{2} W_{n} \geq 0$. By taking the summation of both sides of (13) from $n$ to $n+\rho, \rho<l$, it follows that

$$
\begin{gathered}
\sum_{i=n}^{n+\rho} \Delta W_{i+1}-\Delta W_{i}=\sum_{i=n}^{n+\rho}\left(r_{i}-q_{i-l+k}\right) G\left(y_{i-l}\right)-\Delta W_{n} \\
\geq \beta_{1} \sum_{i=n}^{n+\rho}\left(r_{i}-q_{i-l+k}\right) y_{i-l}
\end{gathered}
$$

From (4) we get

$$
\begin{aligned}
y_{n}=W_{n}+p_{n} y_{n-m} & +\sum_{j=n}^{\infty} \sum_{i=j-l+k}^{j-1} q_{i} G\left(y_{i-k}\right)+F_{n} \\
& \geq W_{n}+F_{n} \\
y_{n} & >W_{n}-\varepsilon, \varepsilon>0
\end{aligned}
$$

Since $\varepsilon$ is arbitrary, it follows that

$$
y_{n} \geq W_{n}
$$

Substituting the last inequality in (16) we obtain

$$
-\Delta W_{n} \geq \beta_{1} \sum_{i=n}^{n+\rho}\left(r_{i}-q_{i-l+k}\right) W_{i-l}
$$




$$
\begin{gathered}
-\Delta W_{n} \geq \beta_{1}\left(\sum_{i=n}^{n+\rho}\left(r_{i}-q_{i-l+k}\right)\right) W_{n+\rho-l} \\
\Delta W_{n}+\beta_{1}\left(\sum_{i=n}^{n+\rho}\left(r_{i}-q_{i-l+k}\right)\right) W_{n-(l-\rho)} \leq 0
\end{gathered}
$$

By Theorem 1-i and in virtue of (12), it follows that the last inequality cannot have eventually positive solution, which is a contradiction.

\section{Conclusion}

1. In this paper we used two series $w_{n}$ and $W_{n}$, and obtained necessary and sufficient conditions for every solution of neutral difference equation of second order with positive and negative coefficients, to be oscillates or tends to infinity as $n \rightarrow \infty$.

2. In condition $\left(\mathrm{H}_{3}\right)$ we can use $\lim _{n \rightarrow \infty} F_{n}=\mathrm{a}$, where a is constant and the results remain true.

3. The conditions $\left(\mathrm{H}_{4}\right)$ and $\left(\mathrm{H}_{5}\right)$ can be improved, and established new conditions.

\section{References}

[1] S. N. Elaydi: An introduction to difference equations. Springer-Verlag New York, Inc., 2005.

[2] E. George Chatzarakis, G. Christos Philos, P. Ioannis Stavroulakis: On the oscillation of solutions to linear difference equation with variable delay, Electronic Journal of Differential Equations, Vol. (2008), No. 50, 1-15.
[3] I. Gyori, G. Ladas: Oscillation theory of delay differential equations with applications. Oxford Clarendon Press, 1991.

[4] Hasan ögunmez, Özkan Öcalan: Oscillations of difference equations with several positive and negative coefficients. Fasciculi mathematic, 51(2013), 115-122.

[5] G. Ladas: Oscillations of difference equations with positive and negative coefficients, Rocky Mountain J. of Math., 20(4) (1990), 1051-1061.

[6] R. N. Rath, L. N. Padhy: Necessary and sufficient conditions for oscillations of solutions of a first order forced nonlinear difference equation with several delays. Fasc. Math., 35(2005), 99-113.

[7] R. N. Rath, L. N. Padhy: Oscillations and non-oscillations of neutral difference equations of first order with positive and negative coefficients. Fasc. Math., 37(2007), 57-65.

[8] X. H. Tang, J. S. Yu, D. H. Peng: Oscillations and nonoscillations of neutral difference equations with positive and negative coefficients. Computers and mathematics application, 39 (2000), 169-181.

[9] A. K. Tripathy, S. Panigrahi: Oscillations in nonlinear neutral difference equation with positive and negative coefficients: International Journal of Difference Equations. Vol. 5, No. 2, (2010), 251-265.

[10] Xiaohui Gong, Xiaozhu Zhong, Jianqiang Jia, Rui Ouyang, Hongqiang Han: Oscillations of first order neutral difference equations. Modern Applied science, Vol. 3, No. 8, (2009), 9094.

[11] Zhiguo Luo, Jianhua Shen: New Oscillation Criteria for Delay Difference Equations. Journal of Mathematical Analysis and Applications, 264 (2001), 85-95.

[12] W. R. Wade: An Introduction to Analysis. Third edition, Pearson Prentice Hall, 2004. 